\title{
Strong GC and AT Skew Correlation in Chicken Genome
}

\author{
Xuegong Deng ${ }^{1}$, Xuemei Deng ${ }^{2}$, and Ilkka Havukkala ${ }^{3}$ \\ ${ }^{1}$ Northeastern university, College of Science, Shenyang, China \\ dengxuegong@tom.com \\ ${ }^{2}$ Department of Animal Genetics and Breeding \& National Key Lab, China Agricultural \\ University, Beijing 100094, China \\ deng@cau . edu. cn \\ ${ }^{3}$ Auckland University of Technology, Knowledge Engineering and Discovery Research \\ Institute, Auckland, New Zealand \\ ilkka.havukkala@aut.ac.nz
}

\begin{abstract}
Chicken genome AT and GC skews for individual chromosomes were visualized simultaneously using a novel method of 2-dimensional colorcoded pixel matrix. The visualizations were compared to those of human, mouse and possum genomes. A strikingly strong correlation of AT skew and GC from small to large scale in chicken genome was found, compared to the other vertebrates. Some local skew correlations were also found for the other vertebrates, but only in small genomic scale. Quantitative measures of correlation were developed, and confirmed the special characteristic of chicken chromosomes. Possible explanations for uniqueness of birds in this respect are discussed. The phylogenetic distribution and evolutionary pressures responsible for this previously unreported skew correlation warrant further study.
\end{abstract}

Keywords: AT/GC skew, skew correlation, chicken genome, chromosome, visualization, 2D.

\section{Introduction}

Division of genomes to large-scale segments of low and high GC\% (isochores) has been attributed to natural selection [1], mutational biases, biased gene conversion [2] and recombination [3]. In addition, the nucleotide skews $(\mathrm{A}-\mathrm{T}) /(\mathrm{A}+\mathrm{T})$ and $(\mathrm{C}-$ $\mathrm{G}) /(\mathrm{G}+\mathrm{C})$ can vary locally and seem to be related to gene distribution, transcription direction and the origin of replication and many other important biological properties in bacteria [4] and in mammals [5]. This suggests that genomes at a local scale need frequently to deviate from Chargaff's 2nd rule [6], which states that in the whole genome scale the frequencies of $\mathrm{A}$ and $\mathrm{T}$ are similar in single stranded DNA, as well as the frequencies of $\mathrm{G}$ and $\mathrm{C}$ [7].

Traditionally, CG-skews have been analyzed by using separate cumulative skew diagrams [8]. Recently AT and CG skews have been combined as cumulative total skew (sum of AT and CG skews) over $1 \mathrm{~kb}$ non-overlapping windows, and used for prediction of replication origins in mammalian genomes [5]. Bacterial genomes [9] and organellar genomes [10] show correlations between nucleotide skews but there is little data from higher eukaryote main genomes in this respect. 
Visualizations of GC skew for circular bacterial genomes has been developed $[11,12]$, but these are not suitable to display skew correlation in large eukaryotic genomes. Therefore other better methods are needed for large-scale easy visualization and analysis of AT and CG skews for whole genomes and eukaryotic chromosomes. We develop here a new method of 2-D color display to show simultaneously both AT skew and GC skew at varying scales in whole chromosomes. Correlation of the skews is easily seen as symmetry along the diagonal in the $2 \mathrm{D}$ matrix representation.

A new summary parameter is developed for symmetry level (correlation) of AT and $\mathrm{CG}$ skews. The developed new visualization method, called Base Skew Double Triangles (BSDT), and correlation quantitation method facilitate nucleotide skew comparisons between various genomes and at various genomic scales. The BSDT visualization software and related information is available at request from the authors.

The new visualization method and quantitative correlation measures discovered that chicken chromosomes have a strong skew correlation in large scale, in strong contrast to the three higher vertebrates studied (opossum, mouse, human). Possible explanations for this difference are discussed.

\section{Methods}

Nucleotide skews show preponderance of one nucleotide frequency over another, e.g. AT skew $=(\mathrm{A}+\mathrm{T}) /(\mathrm{A}-\mathrm{T})$. They are normally calculated over limited windows, or cumulatively along the sequence. In contrast, here we visualize the AT skew and CG skew of whole chromosomes in a square matrix of pixels at scale of $1024 * 1024$ pixels. The DNA sequence is divided into 1024 equal size windows, $W^{i}$, with window length $l=L / 1024$ where $L$ is the length of the whole sequence. For any pair $\mathrm{m}, \mathrm{n}<1024, \mathrm{~m}<\mathrm{n}$, we define the matrix of subsequences from $W^{m}$ to $W^{n}$ as

$$
D_{m, n}=\bigcup_{i=m}^{n} W^{i}
$$

and the AT and CG skews of $D_{m, n}$ can be denoted as $D_{m, n}^{A T}$ and $D_{m, n}^{G C}$. By defining a RGB color function, we draw two symmetrical pixels $(m, n)$ and $(n, m)$ (at the symmetry position) with color $\operatorname{color}\left(D_{m, n}^{A T}\right)$ and $\operatorname{color}\left(D_{m, n}^{G C}\right)$. When the skew value changes from high negative through zero to high positive value, the color changes from deep blue through blue, pink, black, yellow, green to deep green (see Figure 1a). For simultaneous visualization of both AT and CG skews at all scales, we us a color square consisting of two triangles, separated by the diagonal, the bottomleft for the CG skew and the top-right for AT skew. We call this image of triangles

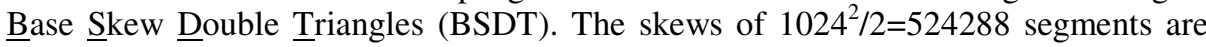
shown in one triangle. Each point in the square represents a base skew of a different subsequence, The changes in AT and GC skews are visualized clearly as color gradients at different scales and locations in the chromosome sequence. Thus, we can clearly observe changes in the skew along the sequence. 

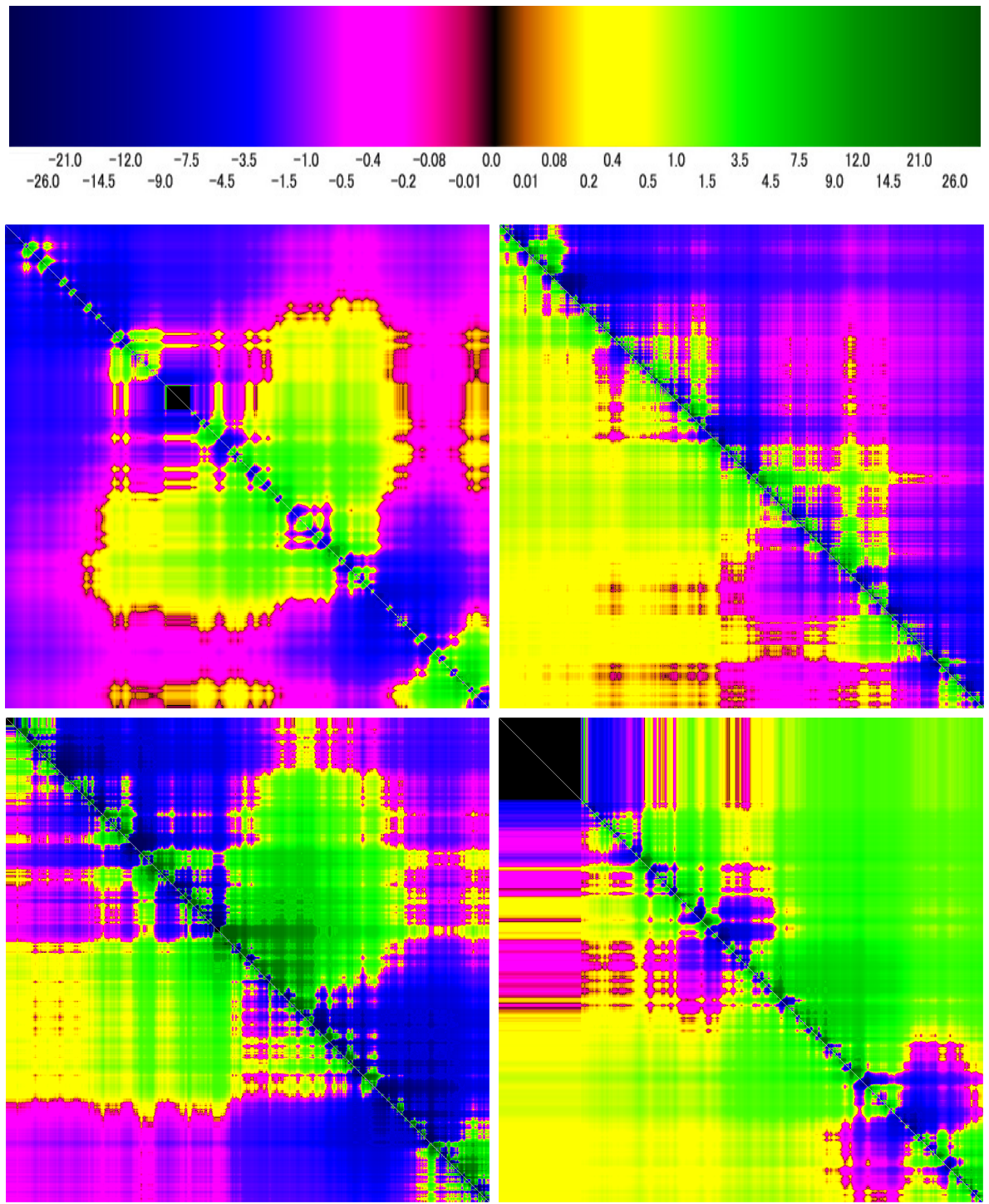

Fig. 1. AT and GC skew correlation visualization in 2D by BSDT. Top bar shows the chromatogram (color scale). Top left: chicken chromosome 8; top right: opossum chromosome 3; bottom left: mouse chromosome 1; bottom right: human chromosome 14 . The lower left triangle represents the AT bias, the upper right triangle represents the GC bias. The clear correlation of AT and CG skews in chicken is evident as symmetry along the diagonal.

By visual observation, most BSDTs of chicken chromosomes are very symmetrical across the diagonal, meaning that the correlation between the AT skew and CG skew is very high for most large chromosomes of chicken at any scale. This visual symmetry phenomenon can be quantified as a summary correlation measure as follows: 
Taking 12 pairs of symmetry lines parallel to the diagonal in the square,

$$
\begin{aligned}
& m-n=\beta \times k+1 \\
& m-n=\beta \times k-1
\end{aligned}
$$

where $\beta$ is the distance between 2 neighboring lines. $\mathrm{k}=0,1,2,3, \ldots$,to 11 in equation (2) and $k=0,-1,-2,-3$, to -11 in equation (3). Since each point pair $(n, m)$ and $(m, n)$ in the lines corresponds two base skews $D_{n, m}{ }^{A T}$ and $D_{n, m}{ }^{C G}$, each line pair has a a set of paired values for each genome sequence. For the 12 correlation coefficients from the 12 paired lines denoted as $C_{i}$, we define the average $C_{i}$ as the correlation level (CL) of the target sequence:

$$
C L_{\beta}^{\text {species_chr* }}=\frac{1}{12} \sum_{i=1}^{12} C_{i}
$$

The species_chr* means for which DNA sequence and $\beta$ means in what scale. Positive values of CL mean positively correlated AT and CG skews, negative values mean negative correlation.

Interpretation of the images is as follows: 1) The skew level of any segment displayed in the BSDT shows strong negative skews as deep blue, changing via black color at no skew to deep green for strong positive skews (see color scale in Figure 1a). 2) The closer the point to the diagonal, the smaller the sequence it represents. 3) The symmetrical color pattern across the diagonal means correlation of GC skew and AT skew of that genome area 4) By comparing the color changes along the diagonal direction we can observe local deviations in skews along the sequence. Note, that in the corners furthest from the diagonal (= largest genomic scale) the skew is less (color is often pink or yellow, that is, closer to black portion in the middle of the color scale).

\section{Data}

The eukaryotic genomic data was selected to compare main branches of higher vertebrates. Species used in this study are shown in Table 2, totaling 87 chromosomes and 9,794 $\mathrm{Mb}$ of sequence data.

Table 1. Genomic data analyzed for AT and GC skew correlation

\begin{tabular}{llll}
\hline Organism & Latin name & Chrom, \# - size (Mb) & Data source \\
\hline chicken & Gallus gallus & $32-984 \mathrm{Mb}$ & UCSC(galGal3) \\
opossum & Monodelphis domestica & $10-3420 \mathrm{Mb}$ & UCSC(monDom4) \\
mouse & Mus musculus & $21-2470 \mathrm{Mb}$ & UCSC(mm6) \\
human & Homo sapiens & $24-2920 \mathrm{Mb}$ & UCSC (hg17) \\
Total & & $87-9794 \mathrm{Mb}$ & \\
\hline
\end{tabular}




\section{Results}

\subsection{AT and GC Skew Correlations}

We generated 2D displays of skew correlation (BSDTs) for 87 chromosomes of 4 fully sequenced species. Representative samples of visualizations are shown in Figure 1. We then calculated the correlation level CL values for $\beta=2,5,10,20,50$ in equations (2) and (3). The CL values of all chromosomes from the four species are listed in table 2, using $\beta=20$. It is remarkable, that chicken chromosomes have clearly the highest symmetry level CL compared with all the other vertebrates.

Table 2. AT and GC skew correlation level CL of ranked chromosomes of four vertebrates, $\beta=20$

\begin{tabular}{|c|c|c|c|c|c|c|c|c|}
\hline Rank & Chicken & CL. & Human & CL. & Mouse & $\mathrm{CL}$ & Opossum & CL \\
\hline 1 & chr8 & 0.984 & chr14 & 0.887 & chrY & 0.730 & chr1 & 0.239 \\
\hline 2 & chr14 & 0.98 & $\operatorname{chr} 15$ & 0.865 & chr1 & 0.718 & chrUn & 0.207 \\
\hline 3 & chr10 & 0.98 & $\operatorname{chr} 7$ & 0.814 & $\operatorname{chr} 12$ & 0.685 & chr6 & 0.050 \\
\hline 4 & chr5 & 0.977 & $\operatorname{chr} 3$ & 0.789 & chr3 & 0.669 & chr8 & -0.023 \\
\hline 5 & $\operatorname{chr} 13$ & 0.975 & $\operatorname{chr} 12$ & 0.780 & $\operatorname{chr} 14$ & 0.632 & $\operatorname{chr} 3$ & -0.251 \\
\hline 6 & chr6 & 0.975 & $\operatorname{chr} 16$ & 0.777 & chr18 & 0.610 & $\operatorname{chr} 2$ & -0.335 \\
\hline 7 & $\operatorname{chr} 4$ & 0.971 & $\operatorname{chr} 20$ & 0.753 & $\operatorname{chr} 13$ & 0.602 & $\operatorname{chr} 4$ & -0.381 \\
\hline 8 & chr19 & 0.97 & chr11 & 0.738 & $\operatorname{chr} 2$ & 0.584 & chr5 & -0.403 \\
\hline 9 & $\operatorname{chr} 7$ & 0.969 & $\operatorname{chr} 5$ & 0.737 & $\operatorname{chr} 10$ & 0.575 & chr7 & -0.445 \\
\hline 10 & $\operatorname{chr} 12$ & 0.967 & chr8 & 0.701 & $\operatorname{chr} 9$ & 0.554 & $\operatorname{chrX}$ & -0.456 \\
\hline 11 & chr11 & 0.967 & $\operatorname{chr} 13$ & 0.698 & chr5 & 0.540 & & \\
\hline 12 & $\operatorname{chr} 20$ & 0.963 & $\operatorname{chr} 9$ & 0.687 & chr16 & 0.513 & & \\
\hline 13 & $\operatorname{chr} 3$ & 0.963 & $\operatorname{chr} 2$ & 0.683 & $\operatorname{chr} X$ & 0.505 & & \\
\hline 14 & chr9 & 0.957 & chr6 & 0.675 & chr6 & 0.498 & & \\
\hline 15 & $\operatorname{chr} 24$ & 0.956 & $\operatorname{chr} 17$ & 0.658 & $\operatorname{chr} 17$ & 0.391 & & \\
\hline 16 & chr1 & 0.955 & $\operatorname{chr} 1$ & 0.658 & $\operatorname{chr} 15$ & 0.377 & & \\
\hline 17 & $\operatorname{chr} 18$ & 0.954 & chr4 & 0.649 & chr19 & 0.370 & & \\
\hline 18 & $\operatorname{chr} 15$ & 0.94 & $\operatorname{chr} 22$ & 0.590 & $\operatorname{chr} 7$ & 0.321 & & \\
\hline 19 & $\operatorname{chr} 23$ & 0.925 & $\operatorname{chr} 18$ & 0.544 & chr4 & 0.232 & & \\
\hline 20 & $\operatorname{chr} 22$ & 0.923 & $\operatorname{chr} X$ & 0.519 & chr11 & 0.218 & & \\
\hline 21 & chrZ & 0.921 & $\operatorname{chr} 10$ & 0.365 & chr8 & 0.181 & & \\
\hline 22 & $\operatorname{chr} 26$ & 0.915 & $\operatorname{chr} 21$ & 0.239 & chrM & -0.030 & & \\
\hline 23 & chr17 & 0.913 & chr19 & 0.110 & & & & \\
\hline 24 & $\operatorname{chr} 2$ & 0.906 & $\operatorname{chrY}$ & -0.252 & & & & \\
\hline 25 & $\operatorname{chr} 21$ & 0.89 & & & & & & \\
\hline 26 & $\operatorname{chr} 28$ & 0.804 & & & & & & \\
\hline 27 & $\operatorname{chr} 27$ & 0.724 & & & & & & \\
\hline 28 & $\operatorname{chr} 25$ & 0.381 & & & & & & \\
\hline 29 & $\operatorname{chr} 32$ & 0.247 & & & & & & \\
\hline 30 & $\operatorname{chr} 16$ & -0.2 & & & & & & \\
\hline 31 & chrM & -0.33 & & & & & & \\
\hline 32 & chrW & -0.38 & & & & & & \\
\hline
\end{tabular}

Since the chromosomes listed in Table 2 have quite different size, but they are represented in a 2D square with same number of pixels, chromosome size might be thought to bias the comparisons. However, we have calculated the CL for many parameter values of $\beta$ for correlation level in many genomic scales, and consistently found that CL for chicken chromosomes were clearly higher than in the other vertebrates for all combinations of species, and almost all chromosomes, using 
parameter combinations $\beta=2,5,10,20,50$ and $\mathrm{n}$ for first 25 chromosomes listed in Table 2. Thus we conclude that chicken chromosomes have higher skew correlation compared to other animals in any scale, based on both visual observation of the BSDT images and the quantitative analysis by CL measure.

One can also compare the chromosomes at the same window scale by a simple visual method. For example, if two DNA sequences have length $30 \mathrm{Mb}$ and $10 \mathrm{Mb}$, then we can compare them by sliding a square 1024/3 in length along diagonal of the BDST of the bigger one, with step 1 . If the longer sequence has the same skew correlation level CL or higher in the smaller window scale, the sliding square should on average will have the same CL or higher compared to the smaller one (see Figure 2).

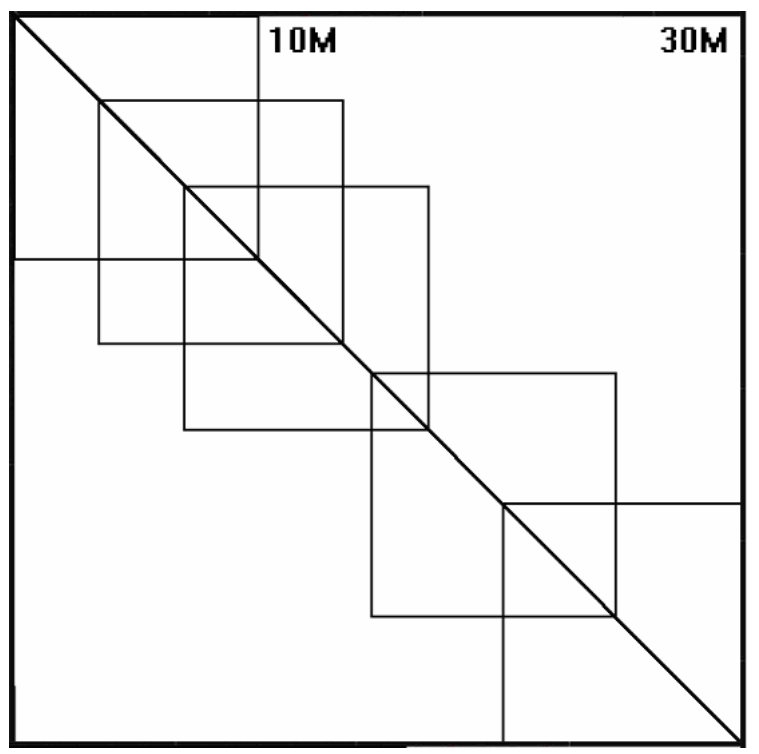

Fig. 2. Sketch map of sliding a smaller square in a bigger BSDT along the diagonal

It is very interesting that we indeed can find some local symmetry segments in other animal's chromosomes with lower CL. For example, human chromosome 2 has some high symmetry local segments found by sliding the square window. Figure $3 \mathrm{a}$ is the BDST of the human whole chromosome 2 and has CL 0.584. that is much smaller than the top ranked chromosomes in Table 2 . We enlarged 3 non-overlapping sliding square windows at length 1024/3, shown in Figure 3d and we can see some local symmetry areas in the chromosome. In certain locations, blue and green color have symmetry along the diagonal, indicating local negative correlation of AT and CG skews. This would lead to a lower CL of the whole chromosome. Unlike birds, other animals show little symmetry in whole genome scale, although many have some symmetry locally. 

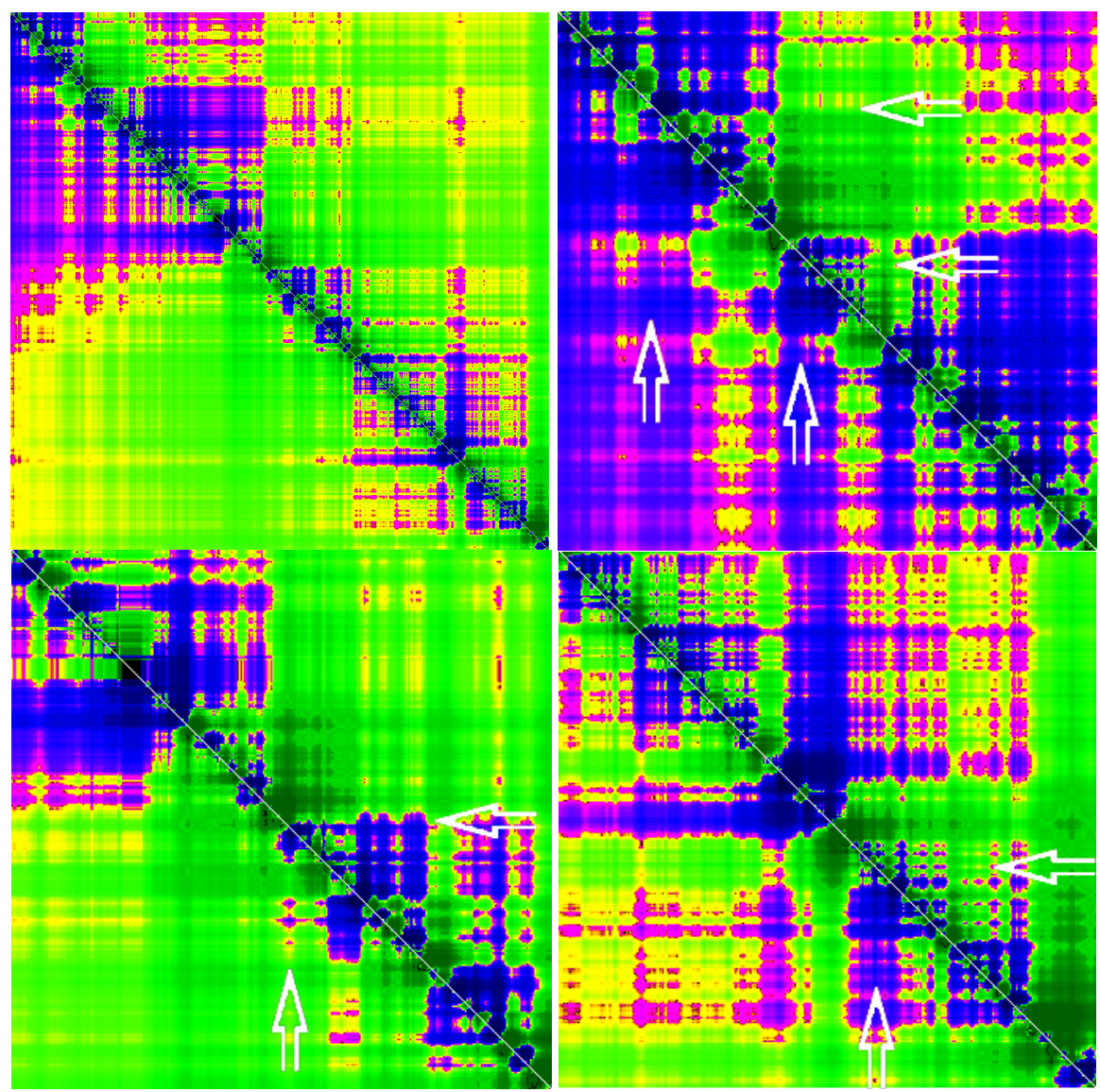

Fig. 3. The BSDT of chromosome 2 of human. Top left: whole chromosome, top right, bottom left and bottom right: successive non-overlapping 1024/3 sliding windows. The white arrows show AT skew and CG skew negatively correlated regions.

\section{Discussion}

In this study, we introduced a new 2D visualization method of BSDT to display and quantify correlation of AT and GC skews in the genome, and discovered a strong correlation in large-scale in the chicken genome. We predict it is prevalent in other bird genomes as well. Further study of the phylogenetic distribution and evolution of this skew correlation awaits access to more genomic data from other bird species and related taxa.

We also discovered local skew symmetries in other animals, which also warrant further investigation. Such smaller scale local skew correlation regions may be related to replication origins at protein coding regions, as shown for human genome (5), or 
other local features tied to transcription activity along the genome. It will be interesting to examine the role and contribution of non-coding and repetitive DNA sequences in this skew correlation. Also the relation between isochore structures and skew symmetry remains to be explored.

Chargaff's second rule is a global rule with many deviations in local genomic scales (4). It must have something to do with double-stranded genome organization, as single-stranded RNA and DNA genomes do not obey the rule (13). Isochore structure may be related to this phenomenon and for such studies the recently constructed human chromosome isochore maps (14) should be useful. It seems established that isochores are declining in current evolution of mammals (15). Webster et al (16) suggest that birds have a history of biased gene conversion, causing and maintaining the isochore structure and more increased divergence in GC-rich regions compared to mammals. As for other sauropsids, only limited reptile data is available, including GC composition in various species measures by analytical centrifugation (17). It would be interesting to see how skew correlation is related to the variation of GC composition at the same genomic scale.

The exact biochemical determinants of evolutionary selection pressures causing and maintaining the special skew correlations reported in this paper are largely unknown, as is their relation to the Chargaff's second rule and isochores. Some possible explanations for the uniqueness of birds are suggested here. Firstly, there may be some unique metabolic aspects in birds, compared to both lizards and mammals, affecting the nucleotide composition. Metabolic constraints and availability of energy in microbes have been shown to affect AT/GC composition (18), as well as environmental factors (19).

Secondly, birds have a higher body temperature and faster metabolism than mammals, due to unidirectional airflow in bird lung and more efficient blood circulation and maximized ATP production (20). Higher temperature and faster metabolism could mean faster mutation rates, affecting biased gene conversion. This could be studied by relating body temperature in various birds and other animals to skew correlation.

Thirdly, chicken genome has been reported to be a mosaic of GC and AT rich isochores (21), though comparisons to reptiles and mammals are still lacking. Isochore structure might affect the biased gene conversion, thus affecting skew correlation.

With more genomic data accumulating from a variety of birds and related taxa we expect interesting findings in this area. The new methods of visualization by BSDT and skew correlation measure CL and their variations should serve as useful tools in this endeavor.

Acknowledgments. This work was funded by the state Major Basic Research Development Program (2006CB102100), National Natural Science Foundation of China (No. 30471233), and National High Technology Research and Development Program of China (No.2001AA222191) for Deng Xuemei. The Auckland University of Technology, Knowledge Engineering and Discovery Institute in Auckland, New Zealand is thanked for facilities for Ilkka Havukkala. 


\section{References}

1. Bernardi, G.: Isochores and the evolutionary genomics of vertebrates. Gene. 241, 3-17 (2000)

2. Eyre-Walker, A., Hurst, L.D.: The evolution of isochores. Nat. Rev. Genet. 2(7), 549-555 (2001)

3. Meunier, J., Duret, L.: Recombination drives the evolution of GC-content in the human genome. Mol. Biol. E. 21(6), 984-990 (2004)

4. Bell, S.J., Forsdyke, D.R.: Deviations from Chargaff's second parity rule correlate with direction of transcription. J. Theor. Biol. 197(1), 63-76 (1999)

5. Touchon, M., Nicolay, S., Audit, B., Brodie, E.-B., d'Aubenton-Carafa, Y., Arneodo, A., Thermes, C.: Replication-associated strand asymmetries in mammalian genomes: Toward detection of replication origins. PNAS 102, 9836-9841 (2005)

6. Chargaff, E.: Some recent studies on the composition and structure of nucleic acids. J. Cell Physiol. 38, 41-59 (1951)

7. Forsdyke, D.R., Mortimer, J.R.: Chargaff's legacy. Gene. 261(1), 127-137 (2000)

8. Grigoriev, A.: Analyzing genomes with cumulative skew diagrams. Nucleic Acids Research 26(10), 2286-2290 (1998)

9. Nikolaou, C., Almirantis, Y.: A study on the correlation of nucleotide skews and the positioning of the origin of replication: different modes of replication in bacterial species. Nucleic Acids Res. 33(21), 6816-6822 (2005)

10. Nikolaou, C., Almirantis, Y.: Deviations from Chargaff's second parity rule in organellar DNA Insights into the evolution of organellar genomes. Gene. 381, 34-41 (2006)

11. Ghai, R., Hain, T., Chakraborty, T.: GenomeViz: visualizing microbial genomes. BMC Bioinformatics 5, 198 (2004)

12. Pritchard, L., White, J.A., Birch, P.R.J., Toth, I.K.: GenomeDiagram: a python package for the visualization of large-scale genomic data. Bioinformatics 22(5), 616-617 (2000)

13. Mitchell, D., Bridge, R.: A test of Chargaff's second rule. Biochem. Biophys. Res. Commun. 340(1), 90-94 (2006)

14. Constantini, M., Clay, O., Auletta, F., Bernardi, G.: An isochore map of human chromosomes. Genome Res. 16(4), 536-541 (2006)

15. Duret, L., Eyre-Walker, A., Galtier, N.: A new perspective on isochore evolution. Gene. 385, 71-74 (2006)

16. Webster, M.T., Axelsson, E., Ellegren, H.: Strong regional biases in nucleotide substitution in the chicken genome. Mol. Biol. Evol. 23(6), 1203-1216 (2006)

17. Hughes, S., Clay, O., Bernardi, G.: Compositional patterns in reptilian genomes. Gene. 295(2), 323-329 (2002)

18. Rocha, E.P., Danchin, A.: Base composition bias might result from competition for metabolic resources. Trends Genet. 18(6), 291-294 (2002)

19. Foerstner, K.U., von Mering, C., Hooper, S.D., Bork, P.: Environments shape the nucleotide composition of genomes. EMBO Rep. 6(12), 1208-1213 (2005)

20. Gao, F., Zhang, C.T.: Isochore structures in the chicken genome. FEBS J. 273(8), 16371648 (2006) 